\title{
Stress-induced electron emission from nanocomposite amorphous carbon thin films
}

\author{
C. H. Poa, ${ }^{\text {a) }}$ R. G. Lacerda, ${ }^{\text {b) }}$ D. C. Cox, and S. R. P. Silva \\ School of Electronics, Computing and Mathematics, University of Surrey, Guildford, \\ Surrey GU2 7XH, United Kingdom \\ F. C. Marques \\ Universidade Estadual de Campinas, Unicamp, Instituo de Fisica "Gleb Wataghin"-13083-970, \\ Campinas-SP, Brazil
}

(Received 4 April 2002; accepted for publication 3 June 2002)

Traditionally, the emission of electrons from materials have been explained using either the FowlerNordheim emission mechanism where high electric fields are used to extract electrons from surfaces or using conventional thermal emission where high currents are used to "boil" off electrons to vacuum. In this letter, we propose an alternative mechanism for electron emission from highly compressive thin films based on stress-induced "band structure" modification of nano-ordered $s p^{2}$ regions in the thin films. Experimental results are recorded which show that the localized compressive stress governs electron emission in the amorphous carbon thin films studied here rather than the surface nanostructures/features or the diamond-like $s p^{3}$ hybridized bond component. This analysis is in agreement with the concept of an internal or nongeometric field enhancement from $s p^{2}$ nanostructures giving rise to high dielectric inhomogeneity within the carbon thin film. The results presented could be extended to explain the anomalous field emission behavior of carbon nanotubes. (C) 2002 American Institute of Physics. [DOI: 10.1063/1.1497442]

Amorphous carbon $(a-\mathrm{C})$ has attracted much attention due to its ability to emit electrons at a relatively low applied electric fields $(<40 \mathrm{~V} / \mu \mathrm{m})$. Comparing this threshold field with conventional flat metal cathodes, electric fields in excess of many hundreds of volts/micron would be required for emission to be initiated. Thus, there is a need for sharp (nanometer scale) cone shaped metal "Spindt" tips ${ }^{1}$ in order to give field crowding and with it electron field emission. Spindt tips, although in use to produce small area displays, are difficult to be produced over very large areas and is an expensive technology to pursue. The capacity for materials such as $a-\mathrm{C}$ to emit at low fields allows for them to be developed as cold cathodes, which can be used for flat panel displays, among other research areas that are technologically important. ${ }^{2}$ Amorphous carbon's unique properties such as chemical inertness, stable mechanical properties, and ease of deposition makes it even more appealing as a candidate for display activities. Various carbon based materials such as amorphous carbon $(a-C),{ }^{3}$ diamond-like carbon, ${ }^{4}$ diamond, ${ }^{5}$ carbon nanotubes, ${ }^{6}$ and carbon containing polymers ${ }^{7}$ have been intensively studied in recent years. However, the precise mechanism responsible for the excellent electron emission of these materials has not yet been fully understood and is presently a subject of intense investigation. Early reports postulated that the emission threshold field $\left(E_{\text {th }}\right)$ is controlled by the diamond-like $s p^{3}$ content based on a negative electron affinity argument. ${ }^{8}$ However, more recent studies ${ }^{9,10}$ have demonstrated that the distribution of the $s p^{2} \mathrm{C}$ sites can be a dominant factor which results in the creation of high

\footnotetext{
${ }^{a}$ Electronic mail: c.poa@eim.surrey.ac.uk

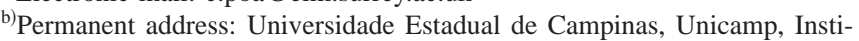
tuo de Fisica "Gleb Wataghin"-13083-970, Campinas-SP, Brazil.
}

internal field enhancement within the $a$-C nanostructure. In a recent letter, Carey et al. ${ }^{9}$ explained the field enhancement of $a-\mathrm{C}: \mathrm{H}$ films in terms of the dielectric inhomogeneities between the $s p^{2}$ and $s p^{3}$ phases. The origin of the field enhancement was explained on the basis of the proximity of the conductive $s p^{2}$ clusters within the film giving rise to electromagnetic field perturbations on a nanometer scale. On the other hand, it is also well known that amorphous carbon films have the unique property of possessing high amounts of intrinsic stress (or internal pressure). In fact, intrinsic stress values $(\sim 12 \mathrm{GPa})$ of almost one order of magnitude higher than those found in other amorphous materials $(<1 \mathrm{GPa})$ such as $a-\mathrm{Si}, a-\mathrm{Ge}$, or metals have been reported. However, although it is known from theoretical studies in the literature that by applying pressure to a material one can modify its electronics properties, e.g., band structure, resistivity, Fermi level, ${ }^{11,12}$ so far, the influence of the internal matrix pressure (or intrinsic stress) on the electron emission properties of any thin film has not been examined. By carefully controlling deposition conditions, the properties of highly $s p^{2}$ rich amorphous carbon thin films which have very different internal pressures (or stresses) (from 1 to about $12 \mathrm{GPa}$ ) are presented in this letter. Due to the graphitic $s p^{2}$ nanoclusters being subject to asymmetric high internal pressure we propose a change in the film band structure, giving rise to the proposed new physical phenomenon of "squeezing" out of electrons from thin films.

The $a$-C films were deposited by an ion beam assisted deposition technique using two Kauffman ion sources. Neon, argon, and krypton gases were introduced in both Kauffman sources, one for the sputtering of a graphite target and the other for the simultaneous assisting (ion bombardment) of the growing film. This enabled independent control of the 


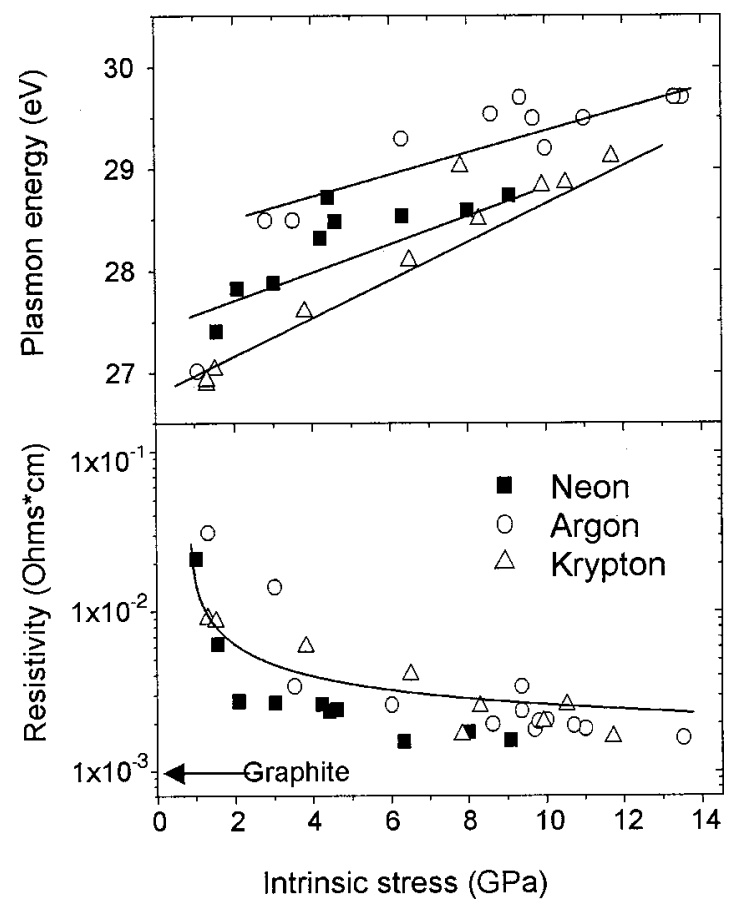

FIG. 1. Dependence of the plasmon energy and resistivity of the $a$-C film as a function of the intrinsic stress with different noble gases $(\boldsymbol{\square})$ neon, $(\bigcirc)$ argon, and $(\triangle)$ krypton.

deposition species and the ion flux used to transfer momentum to the growing film surface. Stress measurements were performed using films deposited on $c$-Si bars, applying an optical bending beam method. ${ }^{13}$ Two laser beams were scanned across the substrate surface to obtain the curvatures of the substrate plus film. The intrinsic stress of the thin film was then calculated using the Stoney equation. ${ }^{14}$ The field emission (FE) characteristics of these films were determined using a sphere-to-plane technique. The threshold field $\left(E_{\mathrm{th}}\right)$ is defined as the macroscopic electric field where an emission current of $1 \mathrm{nA}$ is observed.

The structural analysis of the $a$-C films from x-ray photoelectron spectroscopy (XPS), film density (from Rutherford backscattering), and Raman scattering indicate that the material consists of a highly compressed and dense $s p^{2}$ network. The UV photoelectron spectroscopy (UPS) (top of the valence band) and band gap measurements concur with the view of an absence of a band gap in all the films. ${ }^{13}$ These characteristics are in agreement with the high values of $s p^{2}$ bonds $(\sim 90 \%)$ obtained from EELS, for all the samples. In spite of the highly graphitic nature of the films, there has been an intrinsic correlation between the plasmon energy (from the XPS C $1 s$ plasmon peak) and the resistivity with the intrinsic stress (see Fig. 1). We believe the increase of the internal pressure (high compressive stress) within the graphitic-like $a$-C matrix has led to the increase in the XPS plasmon energy, and a huge decrease of the resistivity. The plasmons, from XPS, can be used as a probe of local valence electronic density of the film. ${ }^{15,16}$ The high values of plasmon energies $(\sim 29.5 \mathrm{eV})$ obtained from XPS are comparable to those values found for highly tetrahedral amorphous carbon ( $t a-C)$ films,${ }^{17}$ and not expected in amorphous thin film with a high degree of $s p^{2}$ bonding. It is also noted that the resistivity, $R_{s}$, of $2 \mathrm{~m} \Omega \mathrm{cm}$ is comparable to the sheet from the substrate for the TEM analysis, some relaxation of
Downloaded 13 Oct 2010 to 131.227 .178 .158 . Redistribution subject to AIP license or copyright; see http://apl.aip.org/about/rights_and_permissions
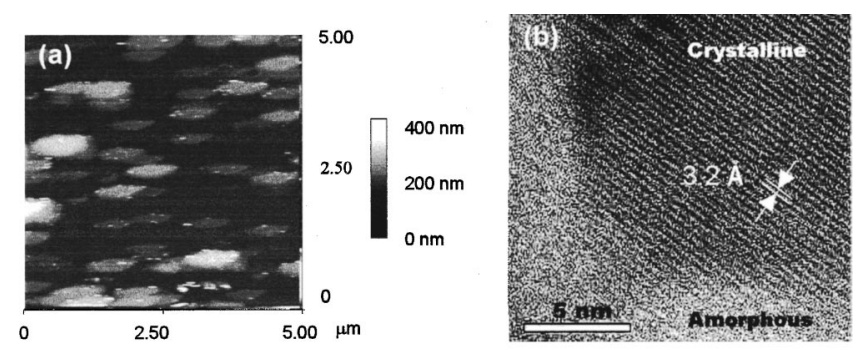

FIG. 2. (a) High resolution STM image of the surface of $a-C$ film grown at an assisting energy of $400 \mathrm{eV}$ with argon as assisting noble gas. Measured at constant current mode, highly conductive nanostructures formed uniformly on the surface. (b) HRTEM illustrating the embedded nanocrystalline particle within an amorphous matrix.

resistance observed in the $c$-axis orientation of crystalline graphite. In addition, the decrease in the resistivity is in agreement with hydrostatic experiments on $a-\mathrm{C}$ films ${ }^{18}$ where it has been shown that an increase in pressure reduces the resistivity. To understand a highly $s p^{2}$ rich $a-\mathrm{C}$ film with a high stress and local electronic density, one has to picture a microstructure that compresses the core/valence electrons and atoms closer together. When the increase in the local density with the internal stress of our films are compared with the internal stress of our films with the decrease of the interplanar distance of microcrystalline graphite, an interplane $s p^{2}$ cluster distance reduction of about $15 \%$ was observed by Lynch et al. ${ }^{19}$ over the same range of pressure. ${ }^{13}$ These results are also in agreement with the recent work of Umemoto et al. ${ }^{20}$ who proposed a new dense, metallic, and rigid form of graphitic carbon with characteristics very similar to the films reported here. The effect of increasing internal pressure can also be quite important on the band structure/density of states of the graphitic $s p^{2}$ films. By having the $s p^{2}$ clusters more close together, the energy gap between $\pi$ and $\pi^{*}$ bands can decrease or in some cases overlap. The increasing overlap of the $\pi$ and $\pi^{*}$ bands can be the main reason for the increasing electron conductivity with pressure. The increasing interaction between the valence electrons is reflected on the high values of electronic density (XPS plasmon energy) shown in Fig. 1(a). The $s p^{2}$ clusters " $c$-axis" spacing is likely to be smaller than that of crystalline graphite and more akin to a 3D graphite network. These clusters would be randomly oriented, with the highly localized strain possibly altering its "band structure," giving rise to a new physical phenomenon that can have beneficial effects on the electron emission process.

The analysis proposed above is further supported by STM and TEM studies. As observed in Fig. 2, there is clear evidence of noncrystalline structures on the surface and in the bulk of the film. The STM images show the presence of highly conductive graphitic nanostructures surrounded by a less conductive region. We attribute this difference to the presence of ordered graphitic nanoparticles embedded in an amorphous carbon matrix. Figure 2(b) shows the nanoparticle fringes with interlayer spacing of $3.2 \AA$ (002) similar to those found in graphite. It is important to observe that, the reduction of the distance between the planes, as proposed above, is only to be expected when the film is under high internal pressure. Therefore, as the films are "peeled off" 


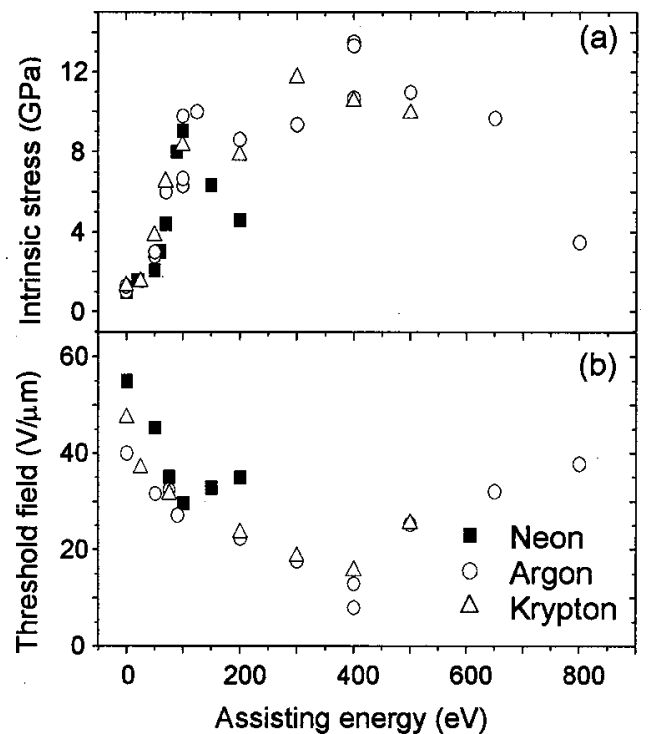

FIG. 3. Variation of threshold electric field and intrinsic stress as a function of the assisting energy with different noble gases $(\boldsymbol{\square})$ neon, $(\bigcirc)$ argon, and $(\triangle)$ krypton. The dependence of the intrinsic stress on the applied threshold field is clear.

the stress occurs and relief the pressure that is exerted on the nanoparticles is expected.

Figure 3 shows the variation of the stress and threshold field of the films with the assisting energy. A remarkable similarity or resemblance between the stress and the threshold field can be observed. Independent of the noble gas used, it is interesting to note that the lowest values of threshold field match the highest stress values in the samples. This provides a clear correlation between the increasing of the internal pressure and the electron emission process. The low threshold FE may be explained by examining the influence of the intrinsic stress on the large and densely populated $s p^{2}$ clusters. It is possible that the stress will induce nongeometric field enhancement within the films by placing the clusters closer together. Recently, Carey et al. ${ }^{9}$ suggested that the field enhancement in $a$-C could be attributed to differences in the dielectric and conductive properties of $s p^{2}$ clusters surrounded by more insulating $s p^{3}$ matrix. The dielectric mismatch between these two phases can have a significant field enhancement if the conductive $s p^{2}$ clusters are closely spaced. The evidence appears to support a field enhancement process based on proximity effects of conductive $s p^{2}$ clusters giving rise to electromagnetic modification of field lines in a highly localized region. It may be postulated that the emission mechanism could be a result of a combination of two effects: (1) the stress, which induces band structure modifications in the films; (2) the local density changes (also a consequence of the internal pressure) which is responsible for the internal field enhancement influencing the absolute value of the threshold field. This work indicates that by the careful control of the stress in nanostructured materials custom designed cathodes can be manufactured and the research opens the doors to a whole new class of cold cathode devices that can be used in the future. Interestingly, recent first principle pseudopotential calculations ${ }^{21}$ show similar density of state modifications to the electronic properties of carbon nanotubes which may explain previously anomalous results when analyzing electron emission using purely a FowlerNordheim approach.

This work was partially sponsored by CNPq, FAPESP. The authors acknowledge Dr. V. Stolojan for helping with the EELS and TEM measurements.

${ }^{1}$ C. A. Spindt, I. Brodie, L. Humphrey, and E. R. Westerberg, J. Appl. Phys. 47, 5248 (1976)

${ }^{2}$ D. Normile, Science 281, 632 (1998).

${ }^{3}$ G. A. J. Amaratunga and S. R. P. Silva, Appl. Phys. Lett. 68, 2529 (1996).

${ }^{4}$ K. Okano, S. Koizumi, S. R. P. Silva, and G. A. J. Amaratunga, Nature (London) 381, 140 (1996).

${ }^{5}$ M. W. Geis, N. N. Efremow, K. E. Krohn, J. C. Twichell, T. M. Lyszczarz, R. Kalish, J. A. Greer, and M. D. Tabat, Nature (London) 393, 431 (1998).

${ }^{6}$ W. Zhu, G. P. Kochanski, and S. Jin, Science 282, 1471 (1998).

${ }^{7}$ I. Musa, D. A. I. Munindrasdasa, G. A. J. Amaratunga, and W. Eccleston, Nature (London) 395, 362 (1998).

${ }^{8}$ B. S. Satyanarayana, A. Hart, W. I. Milne, and J. Robertson, Appl. Phys. Lett. 71, 1430 (1997).

${ }^{9}$ J. D. Carey, R. D. Forrest, and S. R. P. Silva, Appl. Phys. Lett. 78, 2339 (2001).

${ }^{10}$ A. Illie, C. Ferrari, T. Yagi, and J. Robertson, Appl. Phys. Lett. 76, 2627 (2000).

${ }^{11}$ C. Kilic, H. Mehrez, and S. Ciraci, Phys. Rev. B 58, 7872 (1998).

${ }^{12}$ C. Uher, R. L. Hockey, and E. Ben-Jacob, Phys. Rev. B 35, 4483 (1987).

${ }^{13}$ R. G. Lacerda, P. Hammer, C. M. Lepienski, F. Alvarez, and F. C. Marques, J. Vac. Sci. Technol. A 19, 971 (2001).

${ }^{14}$ M. M. de Lima Jr., R. G. Lacerda, J. Vilcarromero, and F. C. Marques, J. Appl. Phys. 86, 4936 (1999).

${ }^{15}$ L. Ley, in Topics in Applied Physics, Vol 56: The Physics of Hydrogenated Amorphous Silicon II, edited by J. D. Jonapoulos and G. Lucovsky (Springer, Berlin, 1984), p. 67.

${ }^{16}$ Y. Katayama, T. Shimada, and K. Usami, Phys. Rev. Lett. 17, 1146 (1981).

${ }^{17}$ P. J. Fallon, V. S. Veerasamy, C. A. Davis, J. Robertson, G. A. J. Amaratunga, W. I. Milne, and J. Koskinen, Phys. Rev. B 48, 4777 (1993).

${ }^{18}$ S. Bhattacharyya and S. V. Subramanyam, Appl. Phys. Lett. 71, 632 (1997).

${ }^{19}$ R. W. Lynch and H. G. Drickamer, J. Chem. Phys. 44, 181 (1966).

${ }^{20}$ K. Umemoto, S. Saito, S. Berber, and D. Tomanek, Phys. Rev. B 64, 193409 (2001).

${ }^{21}$ T. Ito, K. Nishidate, M. Baba, and M. Hasegawa (unpublished). 